\title{
On the possible triple central star system of PN SuWt 2: No ménage à trois at the heart of the Wedding Ring
}

\author{
David Jones $^{1,2 \star} \&$ Henri M. J. Boffin ${ }^{3}$ \\ ${ }^{1}$ Instituto de Astrofísica de Canarias, E-38205 La Laguna, Tenerife, Spain \\ ${ }^{2}$ Departamento de Astrofísica, Universidad de La Laguna, E-38206 La Laguna, Tenerife, Spain \\ ${ }^{3}$ European Southern Observatory, Karl Schwarzschild Strasse 2, 85748 Garching, Germany
}

Accepted $x x x x \operatorname{xxxxxxxx} \mathrm{xx}$. Received $\mathrm{xxxx} \operatorname{xxxxxxxx} \mathrm{xx}$; in original form $\mathrm{xxxx} \operatorname{xxxxxxxx} \mathrm{xx}$

\begin{abstract}
SuWt 2 is a planetary nebula consisting of a bright ring-like waist from which protrude faint extended lobes - a morphology believed to be typical of progenitors which have experienced a close-binary evolution. Previous observations of NSV 19992, the star at the projected centre of SuWt 2, have found it to comprise two A-type stars in a 4.9 day eclipsing orbit, neither of which could be the nebular progenitor. Radial velocity studies provided a hint that the systemic velocity of this double A-type binary might be varying over time, suggesting the presence of a third component hypothesised to be the nebular progenitor. Here, we present an extensive radial velocity monitoring study of NSV 19992, performed with the high-resolution echelle spectrograph UVES mounted on ESO's VLT, in order to constrain the possible variation in the systemic velocity of the A-type binary and its relation to the progenitor of SuWt 2. The observations, acquired over a period of approximately one year, show no evidence of variability in the systemic velocity of NSV 19992. Combining these new observations with previous high-resolution spectroscopy demonstrates that the systemic velocity is also stable over much longer periods and, moreover, is distinct from that of SuWt 2, strongly indicating that the two are not associated. We conclude that NSV 19992 is merely a field star system, by chance lying in the same line of sight as the nebular centre, and that it bares no relation to SuWt 2 or its, as yet unidentified, central star(s).
\end{abstract}

Key words: planetary nebulae: individual: SuWt 2, PN G311.0+02.4 - stars: individual NSV19992 - binaries: close - binaries: eclipsing

\section{INTRODUCTION}

Close-binary interactions are now considered the most-likely mechanism for the formation of aspherical planetary nebulae $(\mathrm{PNe})$, and in PNe where a close-binary nucleus has been detected their shaping influence is clear (Hillwig et al. 2016). However, doubts still remain as to whether central star binarity can be responsible for all observed aspherical $\mathrm{PNe}$, or whether they are responsible for only a fraction of the total population (perhaps those showing the greatest deviation from sphericity). Conservative estimates place the surviving close binary fraction amongst PN central stars at $\sim 20 \%$ (Bond 2000; Miszalski et al. 2009), while the true fraction of PNe that will have experienced some form of binary interaction (including mergers) is almost certainly much higher (de Marco et al. 2004; De Marco et al. 2015). However, given that approximately $25 \%$ of all solar-type

* E-mail: djones@iac.es stars occur in triple or higher order systems (Raghavan et al. 2010), it is possible that such configurations may also be responsible for an appreciable fraction of the PN population (Soker \& Livio 1994; Soker 2016). Based on morphological considerations, Bear \& Soker (2016) reason that the fraction of $\mathrm{PNe}$ originating from triple systems may be as high as $20 \%$ - comparable to the fraction of detectable close binary central stars.

To date, only one PN has been demonstrated to host a triple (or higher-order multiple) star system at its centre, NGC 246 (Adam \& Mugrauer 2014). Here, the orbital separation of the nebular progenitor and its closest companion was wide enough to avoid the common-envelope phase, thus limiting the influence of the triple evolution on the formation of the resulting PN. The central star system of LoTr 5 was concluded to be a hierarchical triple by Jasniewicz et al. (1987) and Malasan et al. (1991). However, a more extensive, data-rich analysis by Van Winckel et al. (2014) found no evidence of radial velocity variations associated with 


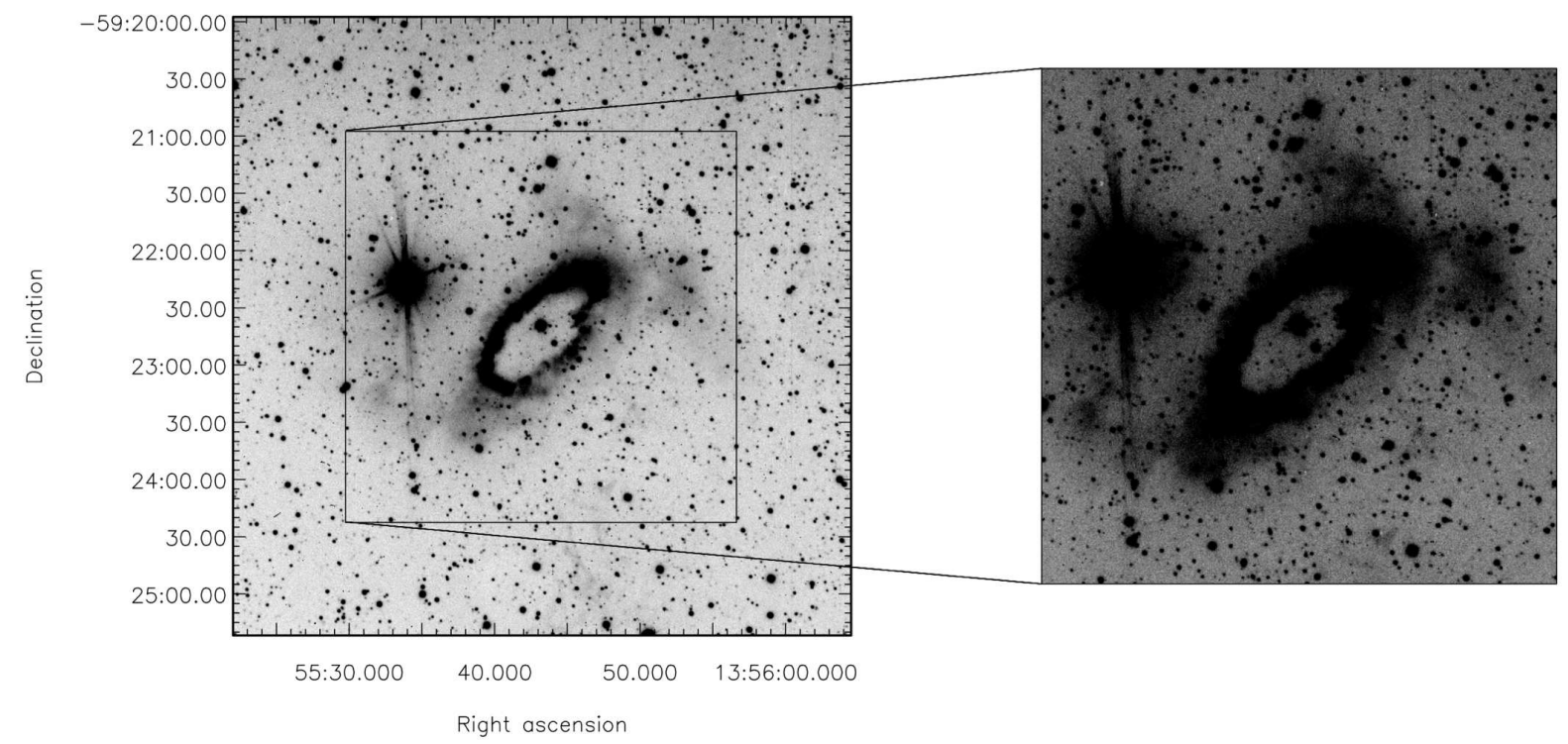

Figure 1. ESO-NTT image of SuWt 2 in the light of [NII]6584 $\AA$ reproduced in part from Jones et al. (2010).

three orbital components, instead finding that the radial velocity variability is due to a wide-binary orbit with a period of a few thousand days. The central star of M2-29 has also been suggested to be a hierarchical triple where the nebular progenitor is in a 23-day period orbit with a close companion and that they in turn orbit a tertiary component with an 18 year period (Hajduk et al. 2008). Miszalski et al. (2011), however, find no evidence for a tertiary component, demonstrating that the observed periodic changes in colour are associated purely with dust obscuration events rather than a wide companion.

The strongest remaining candidate is, without doubt, PN SuWt 2 (Schuster \& West 1976), a ring nebula (see figure 1) where the bright star closest to the projected nebular centre has been shown to be a 4.9 day period binary consisting of two, almost identical, A-type main-sequence stars (Bond et al. 2002; Exter et al. 2003). Exter et al. (2010) performed a detailed analysis of this double A-type system (NSV 19992 from hereon; Kazarovets et al. 1998), showing both components to be $\mathrm{A} 1 \mathrm{~V}$ stars of mass $\sim 2.7 \mathrm{M}_{\odot}$, neither of which could be the source of photoionisation nor the nebular progenitor (the nebular abundances indicate a post-AGB progenitor; Smith et al. 2007). Furthermore, their rotational velocities were found to be different and much slower than expected if the two were tidally locked into rotating synchronously with the orbital period. Exter et al. (2010) argue that this may result from the previous interaction with a currently-unseen post-AGB companion, which is both the nebular progenitor and source of photoionisation, but which was below the detection limit of their UV observations. The most convincing evidence for association between NSV 19992 and the unseen progenitor of SuWt 2 is in the form of apparent variability in the systemic velocity of NSV 19992. Exter et al. (2010) combined spectroscopy from multiple telescopes and instruments taken over a period of nearly 6 years, finding heliocentric systemic velocities rang- ing from approximately -43 to $-4 \mathrm{~km} \mathrm{~s}^{-1}$ (an amplitude of $\approx 20 \mathrm{~km} \mathrm{~s}^{-1}$ around the systemic velocity of the nebula as determined by Jones et al. 2010). They note that (assuming an orbital period of $\approx 0.2$ years, implied by the shortest separation between their radial velocity measurements) the putative third-body in such a system would have an unfeasibly large mass (greater than the Chandrasekhar mass). However, by discarding an outlying measurement (based on low-resolution data), they could revise the radial velocity amplitude down to $\approx 10 \mathrm{~km} \mathrm{~s}^{-1}$, resulting in an estimated mass for the tertiary component of $0.7 \mathrm{M}_{\odot}$. Danehkar et al. (2013) derived a photoionisation model for the nebula and its central star, finding that a central star of mass $\sim 0.7 \mathrm{M}_{\odot}$ could result in the measured nebular line fluxes for a distance (and implied nebular size and post-AGB age) consistent with that determined by Exter et al. (2010) for NSV 19992.

The parallax of NSV 19992 determined by Gaia is 0.655 \pm 0.277 mas (Gaia Collaboration et al. 2016), equivalent to a distance of $0.3-2.7 \mathrm{kpc}$ (5-95\% confidence interval), consistent with the distance determination of Exter et al. (2010) at $D=2.3 \pm 0.2 \mathrm{kpc}$ (found by modelling of the binary). The only distance determination for the PN SuWt 2 based on its nebular properties alone (and not the properties of NSV 19992) is that of Frew et al. (2016) using their $\mathrm{H} \alpha$ surface brightness - radius relation, where an optically thick distance of $3.78 \pm 1.11 \mathrm{kpc}$ was found ${ }^{1}$, only marginally consistent with the distance of the A-type binary NSV 19992 (in spite of the fact that SuWt 2 is used as a calibrator in their determination by assuming that both NSV 19992 is, in fact, related to SuWt 2 and that their common distance

1 Frew et al. (2016) determine a statistical "mean" distance of $3.18 \pm 0.93 \mathrm{kpc}$ for SuWt 2 (more or less consistent with the distance of NSV 19992), but state that, given the properties of the nebula, the optically thick value of $3.78 \pm 1.11 \mathrm{kpc}$ should be used. 
Table 1. A table of measured heliocentric radial velocities versus heliocentric modified Julian date for the two components NSV 19992.

\begin{tabular}{|c|c|c|c|c|}
\hline \multirow{2}{*}{$\begin{array}{l}\begin{array}{l}\text { Modified Julian } \\
\text { date (days) }\end{array} \\
56737.349454\end{array}$} & \multicolumn{2}{|c|}{$\begin{array}{l}\text { Radial velocity of } \\
\text { primary }\left(\mathrm{km} \mathrm{s}^{-1}\right)\end{array}$} & \multicolumn{2}{|c|}{$\begin{array}{c}\text { Radial velocity of } \\
\text { secondary }\left(\mathrm{km} \mathrm{s}^{-1}\right)\end{array}$} \\
\hline & -91.118 & \pm 0.689 & 75.843 & \pm 0.272 \\
\hline 56762.160084 & -110.837 & \pm 0.624 & 94.590 & \pm 0.272 \\
\hline 56773.060922 & -61.900 & \pm 0.651 & 47.622 & \pm 0.296 \\
\hline 56776.139399 & -36.705 & \pm 0.736 & 19.060 & \pm 0.291 \\
\hline 56779.009193 & 68.177 & \pm 0.677 & -85.180 & \pm 0.288 \\
\hline 56784.999457 & 80.965 & \pm 0.760 & -99.180 & \pm 0.257 \\
\hline 56787.004932 & -115.839 & \pm 0.583 & 100.550 & \pm 0.257 \\
\hline 56789.232732 & 96.686 & \pm 0.558 & -113.426 & \pm 0.260 \\
\hline 56792.084737 & -113.517 & \pm 0.574 & 97.896 & \pm 0.267 \\
\hline 56811.025878 & -92.917 & \pm 0.778 & 78.505 & \pm 0.298 \\
\hline 56813.987319 & 99.210 & \pm 0.636 & -117.322 & \pm 0.287 \\
\hline 56831.038256 & -113.780 & \pm 0.704 & 99.735 & \pm 0.313 \\
\hline 56833.993277 & 90.789 & \pm 0.866 & -105.689 & \pm 0.336 \\
\hline 56838.986874 & 83.747 & \pm 0.948 & -100.173 & \pm 0.333 \\
\hline 56841.000608 & -114.474 & \pm 0.743 & 101.932 & \pm 0.329 \\
\hline 56848.117489 & 95.477 & \pm 0.697 & -112.079 & \pm 0.322 \\
\hline 56870.127909 & -107.291 & \pm 0.896 & 92.801 & \pm 0.365 \\
\hline 56874.037974 & 10.415 & \pm 1.507 & -21.500 & \pm 0.335 \\
\hline 56876.069376 & -74.394 & \pm 0.835 & 60.670 & \pm 0.347 \\
\hline 56881.999839 & 55.841 & \pm 0.823 & -71.640 & \pm 0.314 \\
\hline 56885.033362 & -112.847 & \pm 0.745 & 100.015 & \pm 0.338 \\
\hline 56887.018940 & 68.068 & \pm 0.720 & -83.394 & \pm 0.311 \\
\hline 56897.039391 & 86.259 & \pm 0.928 & -100.739 & \pm 0.354 \\
\hline 56903.044846 & 63.119 & \pm 0.766 & -78.646 & \pm 0.293 \\
\hline 57057.366174 & -105.946 & \pm 0.665 & 93.962 & \pm 0.280 \\
\hline 57087.171205 & -76.401 & \pm 0.747 & 64.590 & \pm 0.323 \\
\hline
\end{tabular}

is that determined by Exter et al. 2010). This may be a reflection of the inherent uncertainties in statistical distance determinations for PNe or an indication that NSV 19992 and SuWt 2 are not related. Furthermore, it is interesting to note that NSV 19992 is not at the projected, geometric centre of SuWt 2 (offset by 1"; Smith et al. 2007; Jones et al. 2010), which may be considered evidence that, indeed, the two are unrelated. However, many PN central stars are seen displaced from the geometric centres (e.g. the central star of Abell 41, MT Ser; Jones et al. 2010) and this may be a possible consequence of interactions between the central star components (Soker et al. 1998).

Here, we present a detailed radial velocity analysis of NSV 19992 based on high-resolution spectra taken with the same instrumental set-up (to avoid possible systematics) over a period of almost one year, in order to constrain the possible variability in the systemic velocity of the binary and assess its relationship with PN SuWt 2. The observations and analysis are presented in section 2, while the conclusions are presented in section 3.

\section{OBSERVATIONS AND ANALYSIS}

NSV 19992 was observed 26 times with the Ultraviolet and Visual Echelle Spectrograph (UVES; Dekker et al. 2000) on the Kueyen Unit Telescope of the European Southern Observatory's Very Large Telescope (ESO-VLT) at part of the service mode programme 593.D-0037 between March 212014 and March 62015 (see table 1 for the exact dates of observa-

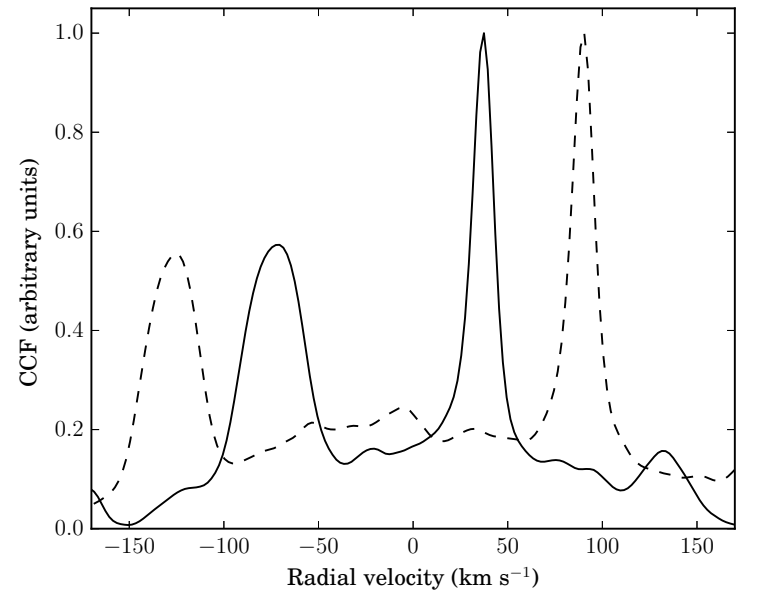

Figure 2. Two example CCFs, corresponding to observations taken at MJD 56773.061 (solid line) and 56787.005 (dashed line), clearly displaying the obvious peaks associated with the two components of NSV 19992. The different peak widths reflect the different rotational velocities of the two components.

Table 2. Orbital parameters

\begin{tabular}{lc}
\hline \hline Parameter & Value \\
\hline$q$ & $0.990 \pm 0.007$ \\
$K_{1}\left(\mathrm{~km} \mathrm{~s}^{-1}\right)$ & $108.23 \pm 0.39$ \\
$K_{2}\left(\mathrm{~km} \mathrm{~s}^{-1}\right)$ & $109.28 \pm 0.31$ \\
$\gamma\left(\mathrm{km} \mathrm{s}^{-1}\right)$ & $-7.60 \pm 0.21$ \\
$P($ days $)$ & $4.9098505^{*}$ \\
$T_{0}($ HJD $)$ & $2456842.7248 \pm 0.0010$ \\
\hline
\end{tabular}

*Fixed parameter taken from Exter et al. (2010)

tion). The instrument set-up was the same for each observation, employing a $0.6^{\prime \prime}$ slit and the dichroic \#1. Simultaneous 1200-s exposures were taken in both arms, with the blue arm centred at $3900 \AA$ (spectral range $~ 3400-4500 \AA$, with the standard HER_5 below-slit filter) and the red at $5800 \AA$ (spectral range $\sim 4800-6800 \AA$, with the standard SHP700 below-slit filter). The resulting data were then processed with the standard ESO pipeline and downloaded from the ESO Science Archive Facility.

The spectra were then continuum subtracted, corrected to heliocentric velocity and cross-correlated against a synthetic A1V spectrum (with the same parameters as those derived by Exter et al. 2010, namely $\mathrm{T}_{\text {eff }}=9250 \mathrm{~K}$ and $\log g=$ 4) produced using the SPECTRUM software (Gray \& Corbally 1994). Each cross-correlation function (CCF) displayed a clear, double-peaked profile, thus allowing us to easily discern the radial velocities of both components of the binary by gaussian fitting of the individual CCFs (example CCFs are shown in figure 2). The resulting heliocentric radial velocities and their associated uncertainties are listed in table 1.

The radial velocities of the two components of NSV 19992 were simultaneously fit in order to check for signs of variability in the systemic velocity, $\gamma$, of the binary. The period was fixed to the value determined by Exter et al. 
(a)

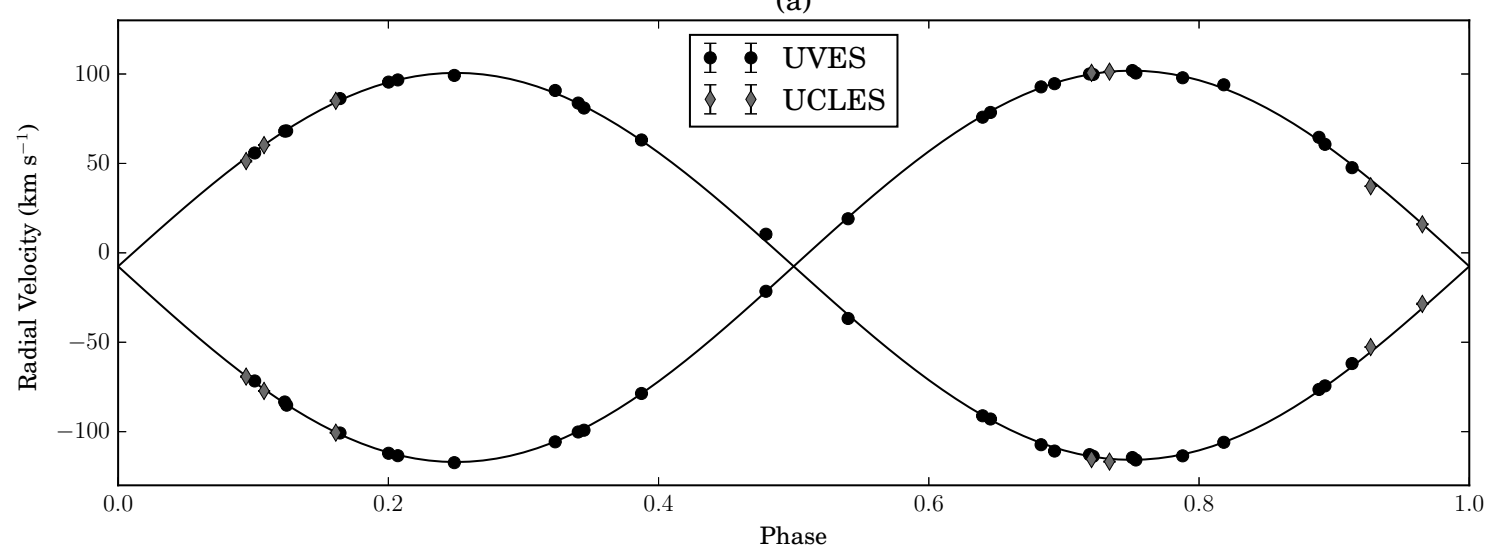

(b)
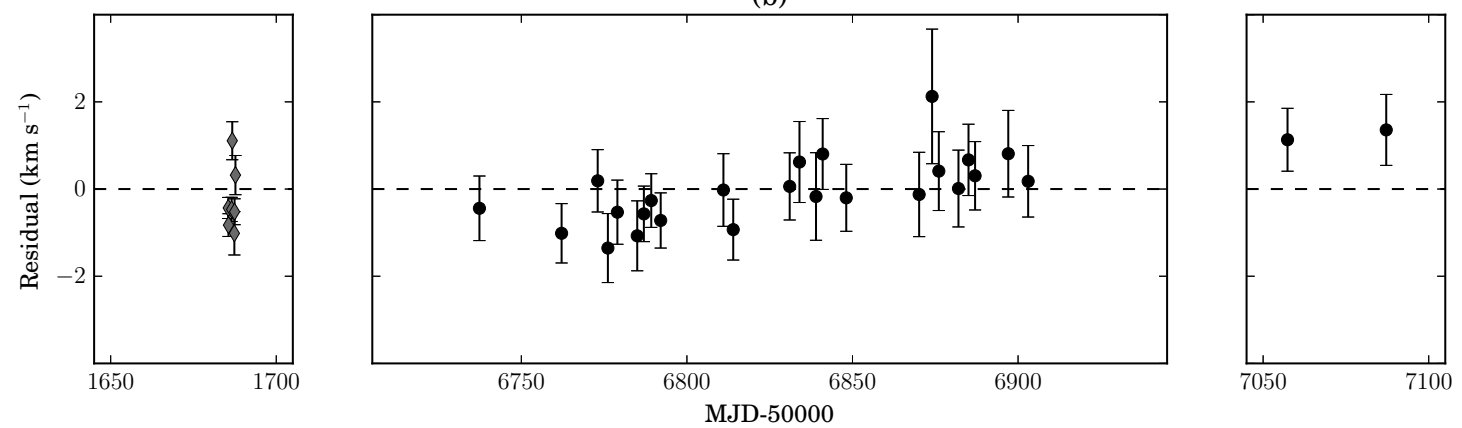

Figure 3. Upper panel: Folded RV curve of NSV 19992 showing a good fit with no appreciable shift in $\gamma$ between epochs (circular filled points are the new UVES data points, while the diamonds are data from AAT-UCLES reproduced from Exter et al. 2010). Lower panel: The residuals for each epoch of UVES and UCLES data (c.f. the sine fit shown in the upper panel).

(2010), who used several epochs of photometric data taken over approximately ten years in order to derive an extremely precise period ( $4.9098505 \pm 0.0000020$ days). The data were satisfactorily fit with a solution containing no drift in systemic radial velocity, with residuals generally of the order of the uncertainty on the radial velocity measurement at each epoch $\left(\sim 1 \mathrm{~km} \mathrm{~s}^{-1}\right)$. The resulting folded radial velocity curve and associated fit is shown in figure 3(a), while the residuals to this fit as a function of MJD are shown in figure 3(b). The parameters of the fit, and the associated derived quantities for NSV 19992 are presented in table 2.

It is immediately clear by looking at the residuals to the fit that our results do not replicate the large, $\sim 20 \mathrm{~km}$ $\mathrm{s}^{-1}$ over a period of $\sim 0.2$ years, amplitude variations in systemic velocity found by Exter et al. (2010). Our data show no signs of any deviation greater than $\sim 2 \mathrm{~km} \mathrm{~s}^{-1}$ over the full observing period ( $\sim 1$ year) implying that the period of any third body (with reasonable mass for a post-AGB stellar remnant) would be of order several years or more (see figure 4). The residuals (figure 3(b)) do appear to show a general upward trend throughout the observing period, however once the uncertainties on the sinusoidal fits are taken into account this trend is not statistically significant (sinusoidal fitting of the residuals results in solutions where the derived amplitudes are less than or approximately equal to the uncertainty of the fit, i.e. a less than $1 \sigma$ correlation). The fit is found to be equally good to the new data presented here as for the AAT-UCLES data of Exter et al. (2010), with residuals $^{2}$ generally $\lesssim 1 \mathrm{~km} \mathrm{~s}^{-1}$ as shown in the left-hand panel of figure 3(b). The agreement is further demonstrated when comparing the derived systemic velocities from our fit to the UVES data $\left(-7.60 \pm 0.21 \mathrm{~km} \mathrm{~s}^{-1}\right)$ and that derived by Exter et al. (2010) when solely considering their AAT-UCLES data $\left(-7.82 \pm 0.41 \mathrm{~km} \mathrm{~s}^{-1}\right)$. It would, indeed, be an extreme coincidence that the only epochs at which the systemic velocity of NSV 19992 has been measured with high-resolution, cross-dispersed echelle data reflect the same phase of the third body's orbit, as such we are led to conclude that the previous radial velocity measurements based on gaussian fitting of single absorption lines in mediumresolution spectra do not accurately reflect the true radial velocity of the system at those epochs. Furthermore, the fact that the residuals to our data (covering almost one year, with

2 Note that the amplitude of the scatter of the UCLES points is more or less the same as the "drift" among the UVES points, further evidence that this apparent, low-level variability is just $\sim 1 \sigma$ deviations about the mean. 
good sampling throughout) are of the same order as to the AAT-UCLES data taken some 13 years earlier, is strongly indicative that the systemic velocity of NSV 19992 is constant on both short and long timescales. Similarly, the constant systemic velocity for both data sets makes a tertiary component in an eccentric orbit rather unlikely. Attempts were made to fit to an eccentric orbit including the data points presented here as well as all data from Exter et al. (2010) in order to evaluate the possibility of a long-period with high eccentricity, however no satisfactory solution could be found. Even selectively excluding data points from lower resolution instruments (for example, just using UVES, UCLES and SAAO data), the long baseline of the (non-variable) UVES data seemingly excludes any eccentric solutions.

In addition to their AAT-UCLES data, the AAT-RGO data from Exter et al. (2010) are also found to agree relatively well with the derived fit (with residuals again of the order of the uncertainties). However, it is important to note that the fit presented here does not reproduce the radial velocities measured by Exter et al. (2010) from their lower resolution SAAO and NTT data. This is perhaps not unexpected given that inclusion of all data (high-, mediumand low-resolution) provided Exter et al. (2010) with a minimum radial velocity semi-amplitude that implied an unfeasibly high central star mass (greater than the Chandrasekhar mass), which led them to ignore the lowest resolution data in estimating their proposed orbital solution. The orbital semi-amplitudes, $K_{1}$ and $K_{2}$, for our fit are systematically lower than those found by Exter et al. (2010) - this can be explained by the different $T_{0}$ we obtain (although our values agree within the uncertainties), resulting in a slight difference in phase for the resulting radial velocity fit. However, the measured mass ratio, $q$, is found to be very close to unity in good agreement with the value determined by Exter et al. (2010). We find no evidence for any deviation from a circular orbit, just as concluded by Exter et al. (2010) from their extensive eclipse timings (while their radial velocity fit did seem to indicate a statistically significant non-zero eccentricity).

\section{CONCLUSIONS}

The systematic velocity of NSV 19992, based on our data and the echelle data of Exter et al. (2010), is not found to vary with time. The residuals to a sinusoidal fit are of order $\lesssim 1 \mathrm{~km} \mathrm{~s}^{-1}$, placing very strong limits on the amplitude of radial velocity variability due to any possible third component to the system. Assuming a reasonable mass for a post-AGB stellar remnant capable of producing and then ionising SuWt 2, any radial velocity variability hidden in the fit residuals would imply an orbital period of several tens of years (see figure 4), certainly not consistent with any kind of common-envelope evolution involving NSV 19992 (therefore greatly limiting the shaping influence of any possible triple configuration). Furthermore, the systemic velocity of NSV 19992, and therefore of any triple system comprised of NSV 19992 and the nebular progenitor (given that large radial velocity variations in NSV 19992 can be ruled out), is significantly different from the systemic velocity of PN SuWt 2 ( -7.6 c.f. $-25 \mathrm{~km} \mathrm{~s}^{-1}$; Jones et al. 2010). This is strongly indicative that the NSV 19992 and SuWt 2 are not

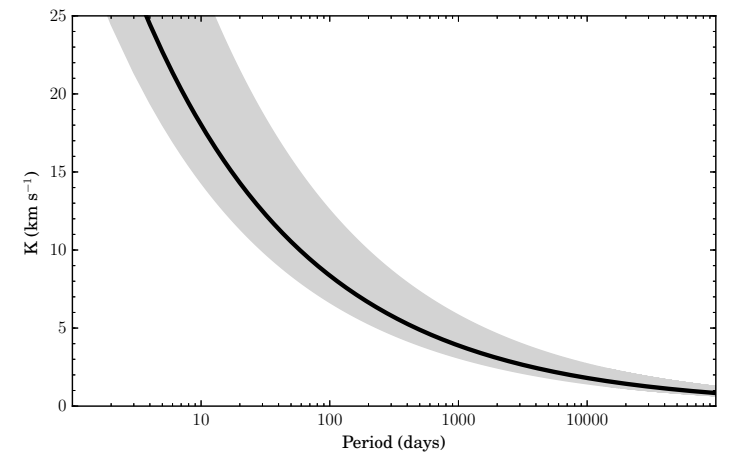

Figure 4. Period versus maximum observed radial velocity $(K)$ for a central star mass, $M_{\mathrm{CS}}=0.64 \mathrm{M}_{\odot}$, as derived by Danehkar et al. (2013) and assuming an inclination of $68^{\circ}$ (Jones et al. 2010) and the most conservative case of a circular orbit. The shaded region encompasses the range $0.5<\mathrm{M}_{\mathrm{CS}}<1.0$ $\mathrm{M}_{\odot}$ to demonstrate the relatively weak dependence on central star mass.

associated as there is little reason to expect that the progenitor star system and resulting PN would have different systemic velocities. The possible discrepancy in distances between NSV 19992 and SuWt 2 highlighted in section 1 adds further weight to the argument that the two are not connected, as does the fact that the morphology of the nebula would be considered more typical of a binary star evolution (Bear \& Soker 2016). As such, we conclude that NSV 19992 is merely a field system and bears no relation to SuWt 2 or its, as yet, unidentified progenitor.

\section{ACKNOWLEDGMENTS}

Based on observations made with ESO Telescopes at the La Silla Paranal Observatory under programme IDs 55.D-0550, 74.D-0373, 593.D-0037. This research has made use of NASA's Astrophysics Data System Bibliographic Services; the SIMBAD database, operated at CDS, Strasbourg, France; the VizieR catalogue access tool, CDS, Strasbourg, France; APLpy, an open-source plotting package for Python hosted at http://aplpy.github.com; Astropy, a community-developed core Python package for Astronomy (Astropy Collaboration 2013); PyAstronomy; SciPy (Jones et al. 2001); NumPy (Van Der Walt et al. 2011). This work has made use of data from the European Space Agency (ESA) mission Gaia (http://www.cosmos.esa.int/gaia), processed by the Gaia Data Processing and Analysis Consortium (DPAC, http://www . cosmos.esa.int/web/gaia/dpac/consortium). Funding for the DPAC has been provided by national institutions, in particular the institutions participating in the Gaia Multilateral Agreement.

\section{REFERENCES}

Adam C., Mugrauer M., 2014, MNRAS, 444, 3459

Astropy Collaboration 2013, A\&A, 558, A33

Bear E., Soker N., 2016, ArXiv e-prints 
Bond H. E., 2000, in Kastner J. H., Soker N., Rappaport S., eds, Asymmetrical Planetary Nebulae II: From Origins to Microstructures Vol. 199 of Astronomical Society of the Pacific Conference Series, Binarity of central stars of planetary nebulae. p. 115

Bond H. E., O'Brien M. S., Sion E. M., Mullan D. J., Exter K., Pollacco D. L., Webbink R. F., 2002, in Tout C. A., van Hamme W., eds, Exotic Stars as Challenges to Evolution Vol. 279 of Astronomical Society of the Pacific Conference Series, V471 tauri and suwt 2: The exotic descendants of triple systems?. p. 239

Danehkar A., Parker Q. A., Ercolano B., 2013, MNRAS, 434, 1513

de Marco O., Bond H. E., Harmer D., Fleming A. J., 2004, ApJ, 602,93

De Marco O., Long J., Jacoby G. H., Hillwig T., Kronberger M., Howell S. B., Reindl N., Margheim S., 2015, MNRAS, 448, 3587

Dekker H., D’Odorico S., Kaufer A., Delabre B., Kotzlowski H., 2000, in M. Iye \& A. F. Moorwood ed., Society of PhotoOptical Instrumentation Engineers (SPIE) Conference Series Vol. 4008 of Society of Photo-Optical Instrumentation Engineers (SPIE) Conference Series, Design, construction, and performance of UVES, the echelle spectrograph for the UT2 Kueyen Telescope at the ESO Paranal Observatory. pp 534545

Exter K., Bond H. E., Stassun K. G., Smalley B., Maxted P. F. L., Pollacco D. L., 2010, AJ, 140, 1414

Exter K. M., Pollacco D., Bell S. A., 2003, MNRAS, 341, 1349

Frew D. J., Parker Q. A., Bojičić I. S., 2016, MNRAS, 455, 1459

Gaia Collaboration Brown A. G. A., Vallenari A., Prusti T., de Bruijne J., Mignard F., Drimmel R., co-authors ., 2016, ArXiv e-prints

Garrison R. F., Hiltner W. A., Schild R. E., 1977, ApJS, 35, 111

Gray R. O., Corbally C. J., 1994, AJ, 107, 742

Hajduk M., Zijlstra A. A., Gesicki K., 2008, A\&A, 490, L7

Hillwig T., Jones D., De Marco O., Bond H., Margheim S., Frew D., 2016, ApJ

Jasniewicz G., Acker A., Duquennoy A., 1987, A\&A, 180, 145

Jones D., Lloyd M., Mitchell D. L., Pollacco D. L., O’Brien T. J., Vaytet N. M. H., 2010, MNRAS, 401, 405

Jones D., Lloyd M., Santander-García M., López J. A., Meaburn J., Mitchell D. L., O'Brien T. J., Pollacco D., Rubio-Díez M. M., Vaytet N. M. H., 2010, MNRAS, 408, 2312

Jones E., Oliphant T., Peterson P., et al., 2001, SciPy: Open source scientific tools for Python

Kazarovets E. V., Samus N. N., Durlevich O. V., 1998, Information Bulletin on Variable Stars, 4655

Malasan H. L., Yamasaki A., Kondo M., 1991, AJ, 101, 2131

Miszalski B., Acker A., Moffat A. F. J., Parker Q. A., Udalski A., 2009, A\&A, 496, 813

Miszalski B., Mikołajewska J., Köppen J., Rauch T., Acker A., Cohen M., Frew D. J., Moffat A. F. J., Parker Q. A., Jones A. F., Udalski A., 2011, A\&A, 528, A39

Raghavan D., McAlister H. A., Henry T. J., Latham D. W., Marcy G. W., Mason B. D., Gies D. R., White R. J., ten Brummelaar T. A., 2010, ApJS, 190, 1

Schuster H. E., West R. M., 1976, A\&A, 46, 139

Smith N., Bally J., Walawender J., 2007, AJ, 134, 846

Soker N., 2016, MNRAS, 455, 1584

Soker N., Livio M., 1994, ApJ, 421, 219

Soker N., Rappaport S., Harpaz A., 1998, ApJ, 496, 842

Van Der Walt S., Colbert S. C., Varoquaux G., 2011, Computing in Science and Engineering, 13, 2

Van Winckel H., Jorissen A., Exter K., Raskin G., Prins S., Perez Padilla J., Merges F., Pessemier W., 2014, A\&A, 563, L10 